\title{
A further biodiversity index applicable to species lists: variation in taxonomic distinctness
}

\author{
K. R. Clarke* ${ }^{*}$ R. M. Warwick \\ Plymouth Marine Laboratory, Prospect Place, West Hoe, Plymouth PL1 3DH, United Kingdom
}

\begin{abstract}
A further biodiversity index is proposed, based on taxonomic (or phylogenetic) relatedness of species, namely the 'variation in taxonomic distinctness' $\left(\right.$ VarTD,$\left.\Lambda^{+}\right)$between every pair of species recorded in a study. It complements the previously defined 'average taxonomic distinctness' $\left(\operatorname{AvTD}, \Delta^{+}\right)$, which is the mean path length through the taxonomic tree connecting every pair of species in the list. VarTD is simply the variance of these pairwise path lengths and reflects the unevenness of the taxonomic tree. For example, a species list in which there are several different orders represented only by a single species, but also some genera which are very species-rich, would give a high $\Lambda^{+}$by comparison with a list (of equivalent $\Delta^{+}$) in which all species tended to be from different families but the same order. VarTD is shown to have the same desirable sampling properties as AvTD, primarily a lack of dependence of its mean value on the sample size (except for unrealistically small samples). Such unbiasedness is of crucial importance in making valid biodiversity comparisons between studies at different locations or times, with differing or uncontrolled degrees of sampling effort. This feature is emphatically not shared by indices related to species richness and also not by properties of the phylogeny adapted from proposals in other, conservation contexts, such as 'average phylogenetic diversity' (AvPD, $\left.\Phi^{+}\right)$. As with AvTD, the VarTD statistic for any local study can be tested for 'departure from expectation', based on a master taxonomy for that region, by constructing a simulation distribution from random subsets of the master list. The idea can be extended to summarising the joint distribution of AvTD and VarTD, so that values from real data sets are compared with a fitted simulation 'envelope' in a $2 \mathrm{~d}\left(\Delta^{+}, \Lambda^{+}\right)$plot. The methodology is applied to 14 species lists of free-living marine nematodes, and related to a master list for UK waters. The combination of AvTD and VarTD picks out, in different ways, some degraded locations (low $\Delta^{+}$, low to normal $\Lambda^{+}$) and the pristine island fauna of the Scillies (normal $\Delta^{+}$, high $\Lambda^{+}$). The 2 indices are also demonstrated to be measuring effectively independent features of the taxonomic tree, at least for this faunal group (although it is shown theoretically that this will not always be the case). The combination of $\Delta^{+}$and $\Lambda^{+}$is therefore seen to provide a statistically robust summary of taxonomic (or phylogenetic) relatedness patterns within an assemblage, which has the potential to be applied to a wide range of historical data in the form of simple species lists.
\end{abstract}

KEY WORDS: Biodiversity · Taxonomic distinctness · Phylogenetic diversity · Sampling properties · Simulation $\cdot$ British marine nematodes

\section{INTRODUCTION}

Species richness measures have traditionally been the mainstay in assessing the effects of environmental degradation on the biodiversity of natural assemblages of organisms. However, the sampling problems associ-

\footnotetext{
*E-mail: b.clarke@pml.ac.uk
}

ated with ascertaining true species richness and making comparable assessments with historical data are well-known, and it should be noted that richness is not the only measurable component of community level biodiversity, even when the data consist simply of lists of species presence/absences. The phylogenetic structure of the assemblage is also clearly important, and an assemblage comprising a group of closely related species must be regarded as less 'biodiverse' than an 
assemblage of the same number of more distantly related species, for example all belonging to different phyla. Measures of phylogenetic structure, based on analysis of cladograms of particular groups of organisms, have been proposed by conservation biologists as a means of assigning conservation priorities that preserve the greatest amount of phylogenetic diversity or 'evolutionary history' (May 1990, Vane-Wright et al. 1991, Williams et al. 1991, Faith 1992, 1994, Humphries et al. 1995, Nee \& May 1997). Little attention, however, has been devoted to analysis of the ways in which environmental degradation affects phylogenetic structure on local or regional scales, and the extent to which properties of this structure can be used as measures of biodiversity for the purposes of biological effects monitoring.

Warwick \& Clarke (1995) introduced the concept of taxonomic distinctness $(\Delta)$ into marine ecology, as a measure of the average degree to which individuals in an assemblage are related to each other. Clarke \& Warwick (1998) examined the statistical sampling properties of measures based both on quantitative species abundances and species presence/absence data $\left(\Delta^{+}\right)$, the latter essentially being a means of comparing non-quantitative species lists. They showed that the mean value of average taxonomic distinctness is independent of sample size or sampling effort-an important advantage over species richness measures-and derived an expression for its theoretical sampling variance. The average taxonomic distinctness $\Delta^{+}$is simply calculated by summing the path lengths through a taxonomic tree connecting every pair of species in the list, and dividing by the number of paths (see Fig. 1a).

Warwick \& Clarke (1998) applied this measure to data on free-living marine nematodes from degraded and non-degraded locations around the British Isles, and provided evidence for a loss of average taxonomic distinctness in locations that were affected by various types of pollution, including sewage, industrial pollution and heavy metal contamination. The index has also been used to compare assemblages of groundfish in NE Atlantic shelf waters (Hall \& Greenstreet 1998, Rogers et al. 1999) and starfish and brittle stars in polar regions (Piepenburg et al. 1997) and the Atlantic (Price et al. 1999). Clarke \& Warwick (1999) looked at various alternatives for how the step lengths between taxonomic levels could reasonably be defined, e.g. proportional to the extent by which the number of taxa decrease at each step, and showed that the results for the simple assumption of constant increments were highly robust. By truncating the taxonomic tree at successive levels, they were also able to determine whether differences in average taxonomic distinctness were due to differences in the higher or lower level structure of the tree.
Another aspect of the phylogenetic/taxonomic structure which, as yet, remains unexplored is the 'evenness' of the distribution of taxa across the tree. Are some taxa over-represented and others under-represented by comparison with what we know of the species pool for the geographical region? This is particularly relevant when comparing biodiversity at larger spatial scales where, in addition to environmental degradation from anthropogenic causes, habitat heterogeneity is likely to influence diversity patterns. For all groups of organisms, specific taxa attain their highest diversity in particular habitats, and if certain habitat types are absent from an area then we might expect some groups of species to be under-represented and others over-represented compared with the regional picture, which could result in a more uneven (more variable) distribution across the tree.

The hypothesis motivating this paper is that, under anthropogenic disturbance, the species that disappear first tend to be those representative of higher taxa which are relatively species-poor. The remaining species are then from a smaller number of groups, each of which tends to be relatively more species-rich (see 'Results and discussion' for some examples of this phenomenon). This effect should manifest itself both as a decrease in average taxonomic distinctness and a decrease in the variability of pairwise relatedness of species in the taxonomic tree. In contrast, one can envisage biogeographic patterns (as above) in which the average taxonomic distinctness might be expected to decrease or remain stable at some locations but the unevenness in phylogenetic structure would increase. Any statistic which measures this variability should ideally have similar sampling behaviour to that of $\Delta^{+}$, i.e. lack of dependence on sample size or sampling effort. In this paper we develop and apply such an index, termed 'variation in taxonomic distinctness', and examine its sampling properties, both in its own right and jointly with average taxonomic distinctness.

\section{DEFINITION OF STATISTICS}

Average taxonomic distinctness (AvTD, denoted by $\Delta^{+}$) is defined by Clarke \& Warwick (1998), from data consisting only of presence or absence of taxa, as

$$
\Delta^{+}=2 \frac{\Sigma \Sigma_{i<j} \omega_{i j}}{s(s-1)}
$$

where $s$ is the number of species present, the double summation is over the set $\{i=1, \ldots s ; j=1, \ldots S$, such that $i<j\}$, and $\omega_{i j}$ is the 'distinctness weight' between species $i$ and $j$. The latter is shown in Fig. 1a as (half) the path length through the standard Linnean tree connecting these species. Typically, equal step lengths $(v)$ 


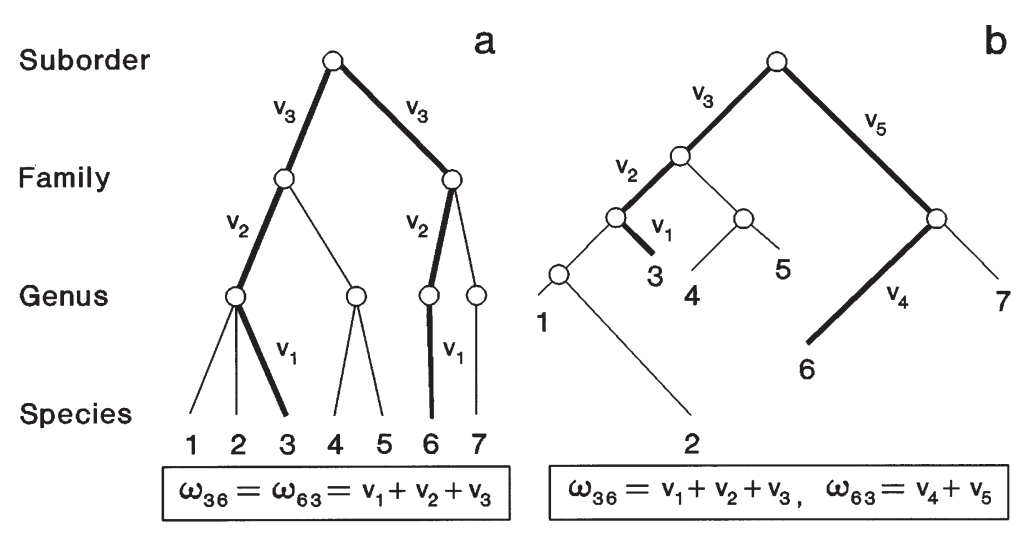

Fig. 1. Examples of (a) taxonomic and (b) phylogenetic trees for a sample of 7 species, and definition of 'distinctness weight' $\left\{\omega_{i j}\right\}$, from species $i$ to $j$, namely the path length through the tree from species $i$ to the first common node with species $j$. A simple mean of these weights defines the 'average taxonomic/phylogenetic distinctness' $\Delta^{+}$of the sample (Eq. 2) and their variance defines the 'variation in taxonomic/phylogenetic distinctness' $\Lambda^{+}$(Eq. 3)

are assumed between each level in the hierarchy (species to genus, genus to family, etc), and Clarke \& Warwick (1999) suggest that they be standardised so that the distinctness of 2 species connected at the highest level (the taxonomically most distant pairing used in a batch of analyses) is set equal to 100 .

It is worth noting in passing that the concept of average taxonomic distinctness is readily extended to a phylogenetic tree whose branch lengths are fully determined (Fig. 1b). The distinctness weight $\omega_{i j}$ is then defined naturally as the distance from species $i$ to the first common node with species $j$. Thus, in Fig. 1b, $\omega_{36}=v_{1}+v_{2}+v_{3}$ and $\omega_{63}=v_{4}+v_{5}$. The asymmetry of the $\left\{\omega_{i j}\right\}$ is then easily catered for in the definition of $\Delta^{+}$by using the more general form:

$$
\Delta^{+}=\frac{\Sigma \Sigma_{i \neq j} \omega_{i j}}{S(s-1)}
$$

This, naturally, is identical to Eq. (1) for a Linnean classification, for which $\omega_{i j}=\omega_{j i}$. Where a phylogenetic tree is fully specified, it clearly comes closer to providing a measurement of evolutionary relatedness or distinctness of 2 species although, in practice, the cruder approximation of a Linnean classification is all that is likely to be available for most groups of marine organisms. This paper therefore focuses only on Linnean classifications, although all results can be straightforwardly generalised.

Average taxonomic distinctness (AvTD) reflects, of course, only one property of a taxonomic structure. It will be effective in contrasting situations in which there are a re- stricted number of higher taxa, for a given number of species, with cases where the same number of species are more taxonomically disparate. It will not, however, distinguish the trees in Fig. 2, which have the same number of species and an identical AvTD but for which the second tree has a more uneven structure across the taxonomic units than the first. The presence of some genera with many species would tend to reduce AvTD, but this could be counterbalanced by the presence of families represented by only 1 (or a very few) species. Such a difference in structure will be well reflected in variability of the full set of pairwise distinctness weights making up the average. Variation in taxonomic distinctness (VarTD, denoted by $\Lambda^{+}$) is therefore defined as

$$
\Lambda^{+}=\frac{\Sigma \Sigma_{i \neq j}\left(\omega_{i j}-\bar{\omega}\right)^{2}}{s(s-1)}=\frac{\Sigma \Sigma_{i \neq j} \omega_{i j}^{2}}{s(s-1)}-\bar{\omega}^{2}
$$

where

$$
\bar{\omega}=\frac{\Sigma \Sigma_{i \neq j} \omega_{i j}}{S(s-1)} \equiv \Delta^{+}
$$

The VarTD statistic is here computed for a range of data sets on soft-sediment marine nematode assemblages in the British Isles, covering polluted and clean environments, island and mainland sites, and diverse habitats.

\section{DATA EMPLOYED}

We have used the same data on marine free-living nematode assemblages from the British Isles as Warwick \& Clarke (1998), for which a comprehensive phy-

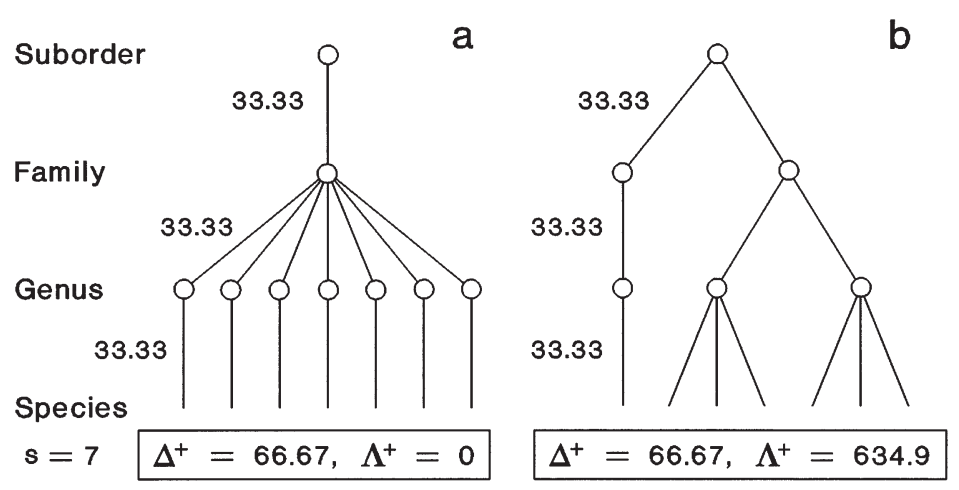

Fig. 2. Two example trees $(a, b)$ for which average taxonomic distinctness $\left(\Delta^{+}\right)$is identical but variation in taxonomic distinctness $\left(\Lambda^{+}\right)$differs substantially, reflecting the greater unevenness of the tree structure in (b) compared with the simple regularity of (a). Note the standardised scaling of path step-lengths such that the maximum distinctness weight $\omega$ attainable for any of the trees being compared is set equal to 100 
logenetic classification based on cladistic principles exists (Lorenzen 1981, 1994) and for which a relatively comprehensive inventory of species is available (Platt \& Warwick 1983, 1988, Warwick et al. 1998). The total number of recorded species in Britain is 395, and these have been classified into genera, families, suborders, orders and subclasses according to Lorenzen (1981, 1994). The data include 14 location/habitat combinations (Fig. 3). For 3 different habitats (sublittoral sediments, intertidal sands and estuarine mudflats) there are examples covering a range of levels and types of pollution:

Sublittoral offshore sediments. N (Fig. 3): the relatively pristine mud and sandy mud at 3 stations off the Northumberland coast (Warwick \& Buchanan 1970); TY: the Tyne sewage sludge dumping ground, a dispersive site where environmental impact on both meiobenthos and macrobenthos, in comparison with appropriate control sites in that region, is relatively slight (Somerfield et al. 1993); L: the heavily industrialised and sewage-polluted Liverpool Bay (Somerfield et al. 1995).

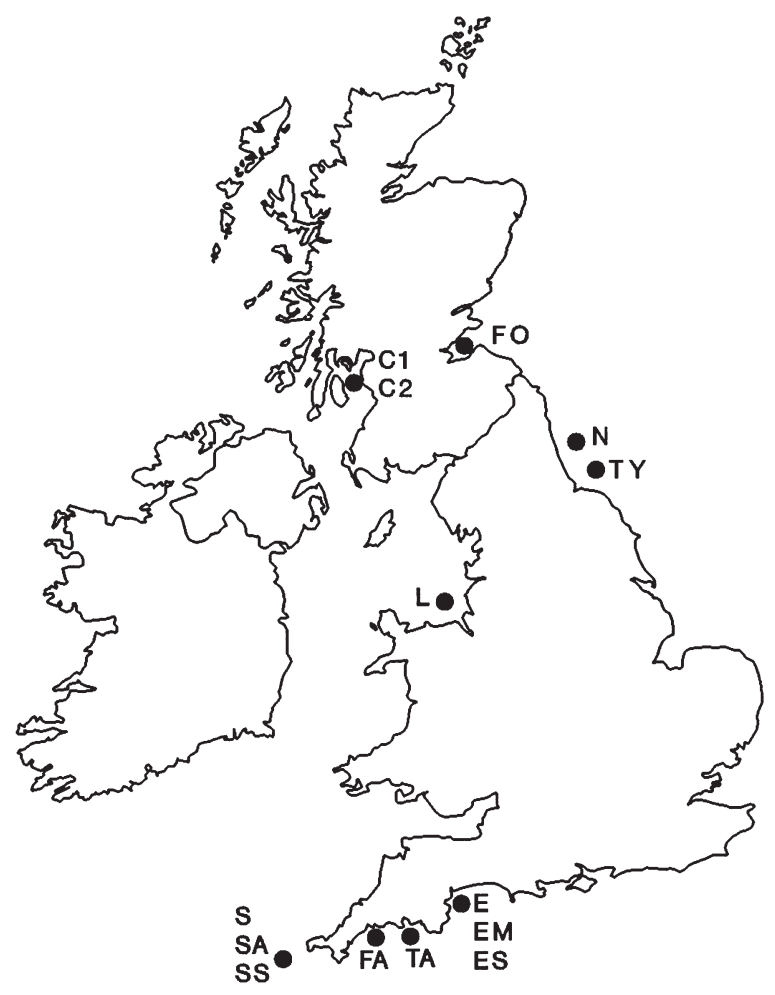

Fig. 3. Map of British Isles showing approximate locations of the studies giving rise to the 14 regional/habitat species lists. S: all Scillies; SA: Scillies algae; SS: Scillies sand; FA: Fal mud TA: Tamar mud; E: all Exe; EM: Exe mud; ES: Exe sand; L: Liverpool Bay; TY: Tyne dumpground; N: Northumberland offshore; FO: Forth sand; C1: Clyde sand (Lambshead study); and C2: Clyde sand (Jayasree study)
Intertidal sand beaches. SS: the pristine sandflats and beaches of the Isles of Scilly (Warwick \& Coles 1977) and ES: the mouth of the Exe estuary (Warwick 1971); the beaches subjected to industrial and sewage pollution, in the Clyde at C1: Ettrick, Irvine and Ayr Bays (Lambshead 1986) and C2: Irvine Bay (Jayasree 1976), and at FO: the Forth (Jayasree 1976). Ettrick Bay is non-polluted according to Lambshead (1986), but since we are considering regional rather than site-bysite concepts, we have included all species he recorded from 16 samples in the 3 bays.

Estuarine intertidal mudflats with reduced salinity. EM: the relatively pristine Exe (Warwick 1971); TA: the Tamar (Austen \& Warwick 1989) with a history of metalliferous mining in the catchment, extensive naval dockyards, and the large conurbation of Plymouth; FA: the Fal estuary where the concentrations of heavy metals in the mud are the highest in the British Isles as a result of a long history of tin-mining (Somerfield et al. $1994 a, b)$.

We have also compared combined species lists for a range of different habitat types from 2 well-defined regions of Britain, namely the Isles of Scilly (S): beach sands with different levels of exposure, intertidal algae (also analysed separately, SA), kelp holdfasts, sublittoral secondary and coarse substrata; and the Exe estuary (E): mudflats in different salinity regimes and at different tidal levels, sand beaches with varying degrees of exposure and grain sizes, and coastal subsoil water from coarse sediments at high water of spring tides. The Liverpool Bay data also arguably come into this mixed-habitat category, since a wide range of sediment types was studied, ranging from fine silts to coarse gravels and stones.

\section{SAMPLING PROPERTIES OF VATTD}

An important characteristic of average taxonomic distinctness is the independence of its mean value from sampling effort, in stark contrast to the estimation of species richness. More precisely, consider a random subsample of $m$ species drawn from the full species set $s(=395$ in the case of the British Isles marine nematode list), and denote the AvTD statistic for that subset by $\Delta_{m}{ }^{+}$. Clarke \& Warwick (1998) show that the mean (in statistical terminology, the expected value, $\left.\mathrm{E}\left({\Delta_{m}}^{+}\right)\right)$of this statistic is always $\Delta^{+}$, the AvTD for the full set of $s$ species, irrespective of $m$. They also derive an expression for the sampling variance, $\operatorname{var}\left(\Delta_{m}^{+}\right)$, as a rather simple function of $m, s$, and 2 variance-related properties of the full tree (see Appendix A). A simple significance test can then be constructed of the null hypothesis that the species list from a particular region/habitat has a taxonomic structure which is representative of 

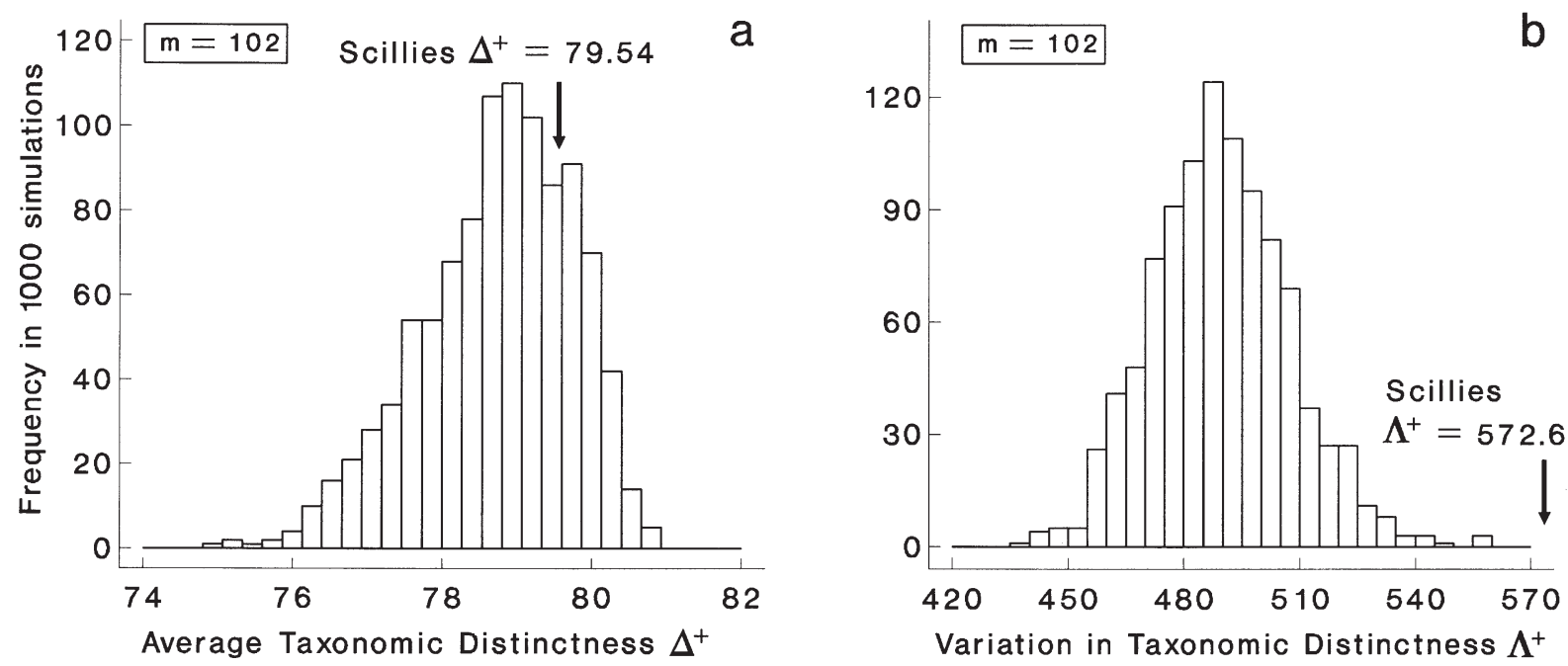

Fig. 4. Histograms of expected distributions of (a) AvTD and (b) VarTD from 1000 simulations. For each simulation, a random subset of $m=102$ species is selected (without replacement) from the full list of $s=395$ marine nematode species recorded from the $\mathrm{UK}$, and the average and variation in taxonomic distinctness $\left(\Delta_{m}{ }^{+}\right.$and $\left.\Lambda_{m}{ }^{+}\right)$computed for that subset. Also indicated are the real values of AvTD and VarTD calculated from the full Scilly Isles data (S), showing no departure from expected $\Delta^{+}$but greater than expected unevenness in taxonomic structure (high $\Lambda^{+}$)

the full biodiversity of the UK list. This holds fixed the observed number $(m)$ of species in the specific regional study (a statistic which is uninformative about the null hypothesis because it reflects simply the differing degrees of sampling effort) and simulates the distribution of $\Delta_{m}{ }^{+}$values expected under the null hypothesis, by making 1000 (say) random selections, each of $\mathrm{m}$ species, from the full UK list. As with any hypothesis test, the true value of $\Delta^{+}$for the specific study is then compared with this null distribution to determine if it lies above or below the critical values, outside of which $5 \%$ of the distribution falls. (Note that Clarke \& Warwick 1998 use the $\operatorname{var}\left(\Delta_{m}{ }^{+}\right)$expression to define approximate limits from a normal distribution, a less computing-intensive operation but one which is not particularly convincing in the case of the $\Delta_{m}{ }^{+}$distribution, with its apparent left-skewness.) The procedure is illustrated in Fig. 4a for the combined Scillies data ( $m=102$ species), and the true value of $\Delta^{+}=79.54$ is seen to fall within the main body of the simulated distribution, implying no departure from the null hypothesis.

Similarly, one can define variation in taxonomic distinctness (VarTD) for a random subsample of $m$ species, denoted by $\Lambda_{m}{ }^{+}$, and Appendix A demonstrates that its expected value is

$$
\mathrm{E}\left(\Lambda_{m}{ }^{+}\right)=\Lambda^{+}-\operatorname{var}\left(\Delta_{m}{ }^{+}\right)
$$

Thus, $\Lambda_{m}{ }^{+}$is not exactly unbiased for $\Lambda^{+}$, the VarTD for the full tree of $s$ species. It is an underestimate since the second term is positive, but the structure of $\operatorname{var}\left(\Delta_{m}{ }^{+}\right)$ given in Appendix A suggests that this bias term will be very small. This is borne out for the British Isles nematode list used to construct Fig. 5b, where the thin continuous line denotes the theoretical mean, $\mathrm{E}\left(\Lambda_{m}{ }^{+}\right)$, as a function of $m$. Whilst for very small $m$ there is a small bias, this quickly tends to zero as $m$ increases. For example, for $m=10, \mathrm{E}\left(\Lambda_{m}{ }^{+}\right)=468.1$ compared with $\Lambda^{+}=$ 490.2 , giving a negative bias of just under $5 \%$, whereas for $m=30$ the bias is only about $1 \%$, for $m=60$ it is $0.4 \%$ and for $m=100$ it drops to about $0.2 \%$. In the context of the generated range of $\Lambda_{m}{ }^{+}$values, for repeated random subsets of $m$ species, the bias is seen to be entirely negligible for all realistic values of $m$. For a particular value of $m(=102)$ this can be seen in Fig. $4 b$, in relation to the 'all Scillies' data, where the simulated distribution under the null hypothesis is clearly centred around the UK average value of $\Lambda^{+}=490.2$, with simulated values ranging from about 440 to 550 . More generally, it is seen from the funnel plot of Fig. $5 b$, where the thick lines indicate the limits within which $95 \%$ of simulated values lie, for a range of $m$.

As with AvTD, the VarTD statistic is therefore seen to have the very desirable sampling property of insensitivity to sampling effort, reflected in the number of species actually observed. This provides a justification for comparing its value for species lists (from the same defined faunal group) over different areas or times, which may have involved collection and analysis by different workers, employing disparate sample sizes. It is implicit that revisions of nomenclature through time are reconciled, also that the different workers do not operate to different standards of taxonomic rigour in identifications (or, if they do, they do not do so in a sys- 

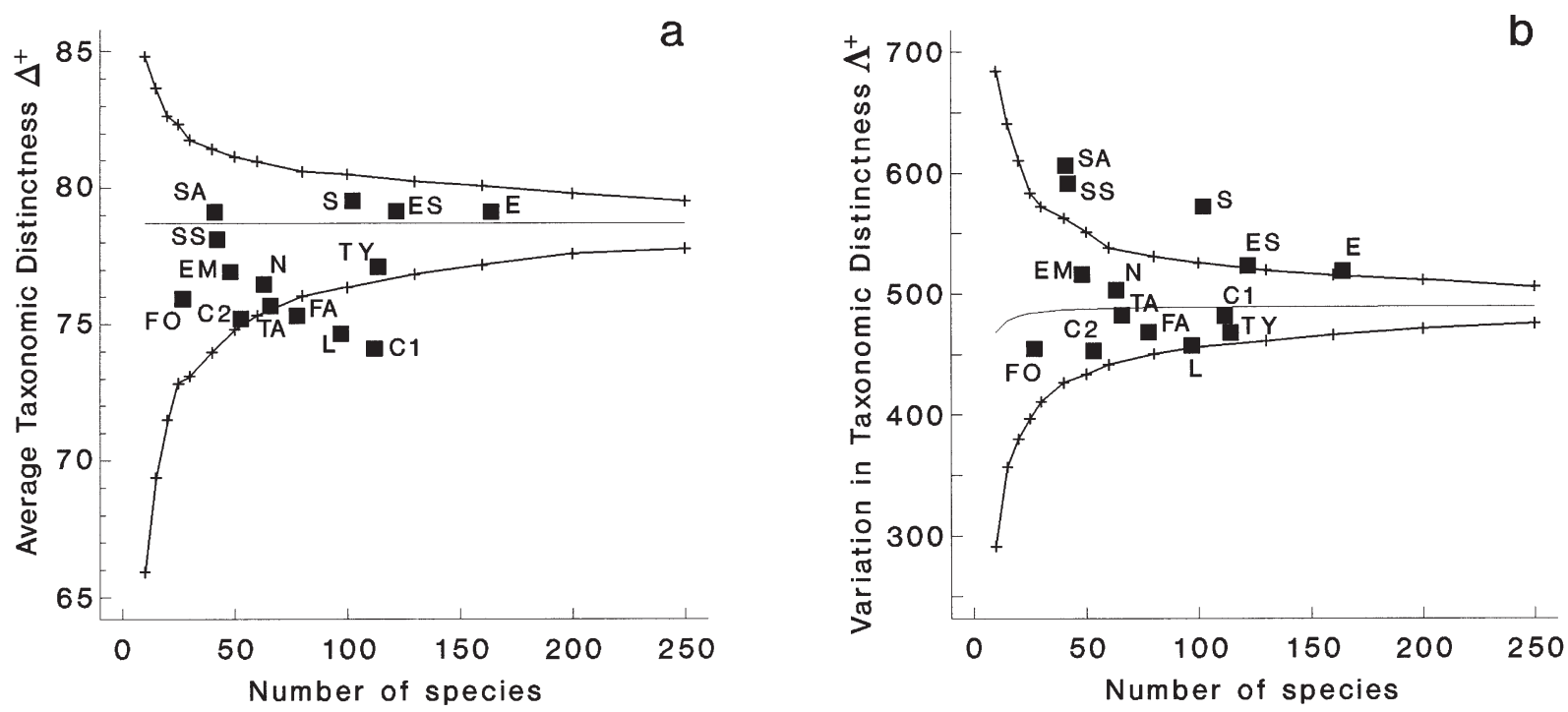

Fig. 5. The $95 \%$ probability funnels (thick line and crosses) for (a) AvTD and (b) VarTD from 1000 independent simulations for each subset size (number of species, $m=10,15,20, \ldots 250$ ) drawn randomly from the 395 UK nematode species. For each specific $m$, $2.5 \%$ of the simulations gave $\Delta_{m}{ }^{+}\left(\Lambda_{m}{ }^{+}\right)$values above the upper, and $2.5 \%$ below the lower limits of these funnels. Thin line denotes the theoretical mean for such random selections, indicating the exact unbiasedness of $\Delta_{m}{ }^{+}$for $\Delta^{+}$(AvTD for the full 395 species), and the near unbiasedness of $\Lambda_{m}{ }^{+}$for $\Lambda^{+}$, irrespective of $m$. Also shown are the actual values of AvTD and VarTD for the 14 regional/habitat species lists, plotted against the number of species in each list. Note the significantly below expectation $\Delta^{+}$values for some degraded locations and significantly above expectation $\Lambda^{+}$values for all the Scilly Isles lists. Abbreviations as in Fig. 3

tematic way, introducing bias). In a general discussion of the utility of measures based on taxonomic distinctness, Clarke \& Warwick (1999) detail such caveats, including the potential dangers of comparing indices across very disparate faunal groups, for which the activities of 'lumpers' on one side, and 'splitters' on the other, could make the designations of taxonomic levels non-comparable. For this reason, the present example confines itself to a fairly coherently-defined group, the marine nematodes.

As an aside, it should be noted that unbiasedness is far from being a universal property of potential biodiversity measures based on the taxonomic hierarchy. For example, Faith (1992) defined a phylogenetic diversity statistic, $\mathrm{PD}$, which corresponds in the current context to the total branch length in the taxonomic tree (e.g. for Fig. 2b, PD = 400). This is essentially a richness measure, since the total branch length must increase each time a new species is added, and practical plots of PD against number of species ( $s$ ) tend to show a simple linear relationship with rather little scatter (e.g. Fig. 6a displays a near-linear relationship for the 14 nematode data sets). However, in a very natural way, PD can be converted to a mean statistic, which parallels $\Delta^{+}$, by defining average phylogenetic diversity (AvPD) as:

$$
\Phi^{+}=\mathrm{PD} / S
$$

This can be thought of as the average amount of phylogenetic diversity (branch length) contributed by a randomly chosen species to the total $\mathrm{PD}$, taking into account how much of this contribution is novel and how much already contributed by other species (Faith describes how to achieve a simple breakdown of the total PD into actual contributions from each species, as part of the process of defining taxa which should attract high conservation status). One might expect $\Phi^{+}$ largely to remove the dependence (in mean value) on the number of species $S$, since the addition of each new species could either increase or decrease the average value $\Phi^{+}$, depending on whether the new species was distantly or closely related, respectively, to the existing species set. This turns out not to be the case, as illustrated in Fig. 6b. Under the same random subsampling model as applied to $\Delta^{+}\left(\right.$and $\left.\Lambda^{+}\right)$, species subsets of fixed size $m$ can be drawn from a master list, for which AvPD for the subset is denoted by $\Phi_{m}{ }^{+}$and for the master list by $\Phi^{+}$. A general expression for $\mathrm{E}\left(\Phi_{m}{ }^{+}\right)$is not mathematically tractable, but a negative bias in estimating $\Phi^{+}$is implicated and, unlike $\Lambda^{+}$estimation, it is not negligible. Fig. 6b shows the simulated mean (thin line) from 1000 subsamples of each size, $m=10,15,20, \ldots$, for the British Isles nematode master list of 395 species, and adds the actual values of $\Phi^{+}$computed for the 14 regional data sets. In sharp contrast with Fig. 5, the dependence of average phylogenetic diversity (AvPD) on the sample size is clear, and it could be highly misleading simply to compare 2 values of $\Phi^{+}$based on different studies with differing degrees of sampling 

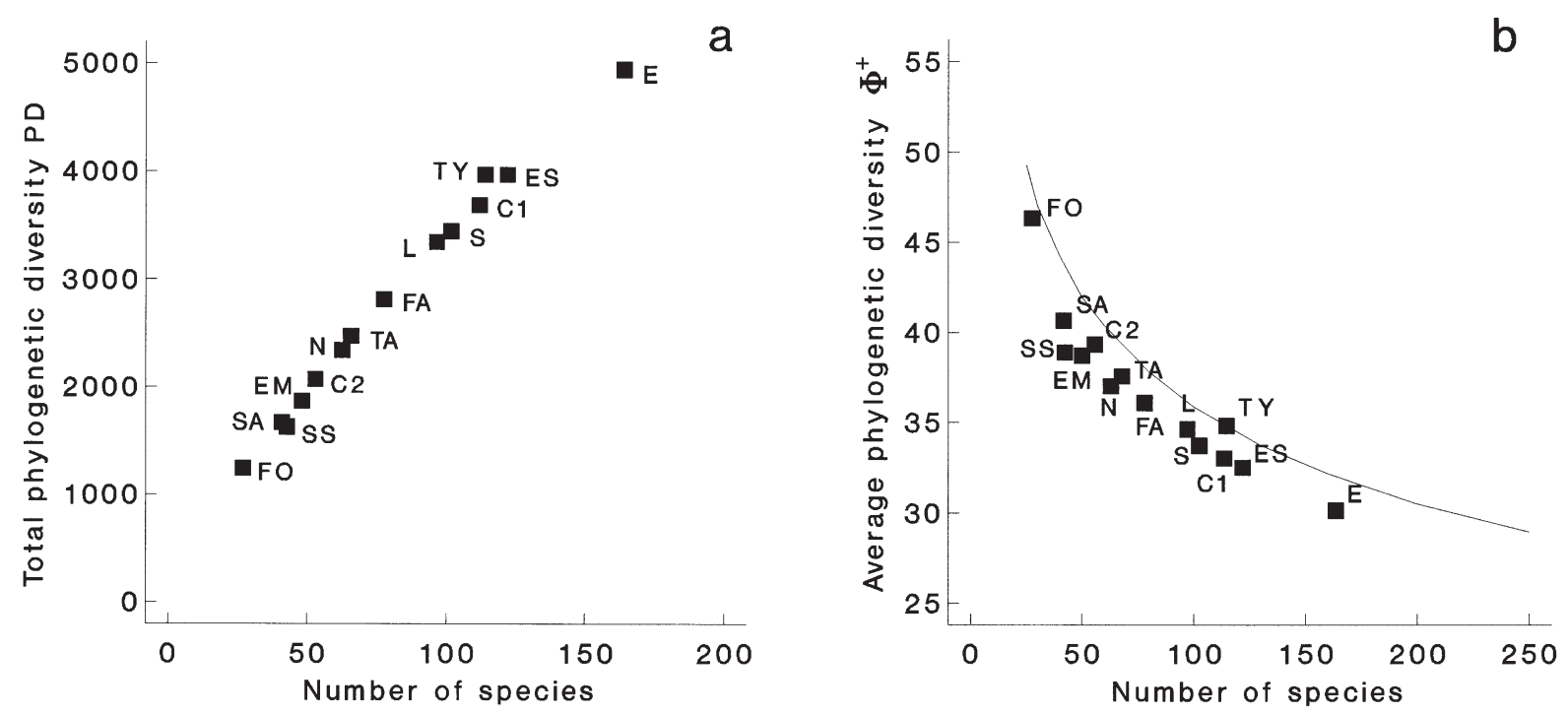

Fig. 6. (a) The (total) phylogenetic diversity (PD), as defined by Faith (1992), computed here as the total branch lengths of the taxonomic (rather than phylogenetic) trees for each of the 14 regional/habitat species lists of UK marine nematodes. These total PD values are plotted against the number of species in each list, demonstrating a near-linear relationship. Attempting to remove this dependence by dividing PD by the number of species in the list gives (b) average phylogenetic diversity, $\Phi^{+}$, for each of the 14 data sets. A strong (negative) dependence on list size remains, as is clear also from the mean (thin line) from sets of 1000 random simulations of species subsets of size $m(=10,15, \ldots 250)$, drawn from the full list of 395 UK nematodes. The contrast with Fig. 5 is stark and shows the difficulty of comparing AvPD values between studies with differing sampling effort. Abbreviations as in Fig. 3

effort. Whilst one could attempt to compensate for this bias when working with a well-defined species pool (e.g. in Fig. 6 b by relating the $\Phi^{+}$value for each data set to its calculated expectation under the random subsampling mechanism) this now depends heavily on appropriate definition of that underlying species pool, which is not the case at all for the $\Delta^{+}$and $\Lambda^{+}$statistics. For AvTD and VarTD, the lack of dependence (in mean value) on sampling effort can be seen as validating direct comparison of calculated values, irrespective of the existence or definition of a 'master list'.

\section{RELATIONSHIP OF VarTD TO AvTD}

Returning to $\Delta^{+}$and $\Lambda^{+}$, with their desirable lack of dependence on sampling effort, the next practical question concerns the extent to which they encapsulate independent information about the taxonomic hierarchy. At one extreme, if they are deterministically linked, the calculation of VarTD in addition to AvTD would add no useful information; at the other extreme, they may reflect entirely unrelated aspects of the taxonomic structure. The reality will be within these extremes, but will depend on the particular context: few general results can be anticipated here. In standard statistical computations there is an orthogonality between variance and mean calculations, but such models do not carry over to the current situation where the weights $\left\{\omega_{i j}\right\}$ for the observed species pairs are not independent of each other.

One theoretical result that can be demonstrated is for the simple case where there are only 3 levels in the hierarchy: species within genera within a single family. Under the usual standardisation, in which path lengths between species in the same genus are set to 50 and between species in different genera to 100 , it is demonstrated in Appendix B that $\Delta^{+}$and $\Lambda^{+}$are perfectly dependent:

$$
\Lambda^{+}=\left(100-\Delta^{+}\right)\left(\Delta^{+}-50\right)
$$

This is a quadratic relationship which goes to zero in the 2 extreme cases of all species in 1 genus $\left(\Delta^{+}=50\right)$ or all species in separate genera $\left(\Delta^{+}=100\right)$. In practice though, for a compressed tree with only 3 levels, there will be multiple genera with several species in many of them, and $\Delta^{+}$will take values much nearer the upper than the lower part of this range. The relationship of $\Lambda^{+}$ to $\Delta^{+}$will therefore be approximately linear, with a negative correlation close to -1 .

Such simple dependence will quickly break down for a more realistic, richer hierarchy, at a greater number of levels. Any mechanistic dependence between $\Lambda^{+}$ and $\Delta^{+}$in a specific context, e.g. for the 7 levels of the master list of British Isles nematodes, can again be explored by selecting random subsets of fixed size $m$ from the full set of $s$ species. Utilising the same simulations as underlie the funnel plots in Fig. 5, a series of 


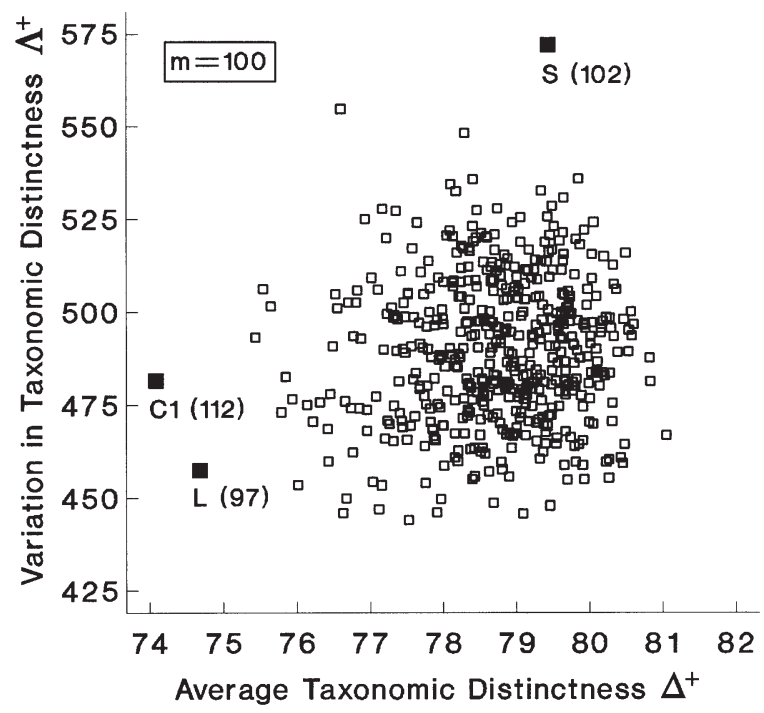

Fig. 7. Scatter plot of VarTD against AvTD values from 1000 simulations (only 500 are plotted, for clarity) of $m=100$ species drawn randomly from the full list of 395 UK nematodes, showing that the 2 statistics will provide practically independent summaries of the taxonomic structure in this case. Also shown (ם) are the actual AvTD, VarTD pairs for the Clyde 1 (C1), Liverpool Bay (L) and 'all Scillies' (S) data sets, which contain approximately 100 species (exact number in parentheses) and whose values are seen to fall outside the range of any realistically constructed envelope for the simulations

scatter plots can be created of simulated $\Lambda_{m}{ }^{+}$against $\Delta_{m}{ }^{+}$values for each specific $m$. Fig. 7 displays just one of these, for $m=100$; each point is the result of a single random draw (without replacement) of 100 species from the 395 in the full nematode list. The lack of a sizeable correlation between $\Lambda_{m}{ }^{+}$and $\Delta_{m}{ }^{+}$is immediately apparent and the same lack of dependence is also found for other values of $m$. Table 1 gives the correlation coefficients between $\Lambda_{m}{ }^{+}$and $\Delta_{m}{ }^{+}$for subsets of size $m=10,15,20, \ldots$ and these are all seen to be smaller than 0.1. (Note that although 1000 simulated values are generated for each $m$, in Fig. 7 these are pruned to display only every other point along the $x$ axis, purely for reasons of clarity of the resulting plot. All computations, however, such as the correlations presented in Table 1 and the model fitting of the later section, are calculated on the full sets of 1000 random simulations.)
The implication in this case is that computation of $\operatorname{VarTD}\left(\Lambda^{+}\right)$is adding fresh summary information about the taxonomic relationships in a sample to that provided by $\operatorname{AvTD}\left(\Delta^{+}\right)$, and the conclusions for the 14 regional/habitat nematode lists are examined later. Note, however, that there is no guarantee that this approximate independence of the 2 measures of taxonomic distinctness will carry over to classification trees for other faunal groups or regions of the world. In fact, preliminary indications are that these innate correlations can be either substantially negative or positive, as well as approximately zero, in other contexts. This does not invalidate the use of both measures of course (it is commonplace to calculate standard suites of diversity indices, such as Shannon, Pielou's evennness, Simpson etc., which are certainly highly interdependent) but it does suggest that they are best viewed in combination, in relation to their joint distribution, as simulated in plots such as those of Fig. 7.

\section{RESULTS AND DISCUSSION}

Fig. 5a displays the $95 \%$ funnel for the simulated distribution of average taxonomic distinctness for random subsets of fixed size $m$ from the UK marine nematode list; that is, $2.5 \%$ of the simulated values fall below the lower limit and $2.5 \%$ above the upper limit for each $m$. Superimposed on this are the true values of AvTD $\left(\Delta^{+}\right)$calculated from species lists for each of the 14 region/habitat combinations described earlier (Fig. 3). Warwick \& Clarke (1998) point out the tendency for $\Delta^{+}$to take lower than expected values for the 'polluted' sites, in some cases falling below the lower limit of the funnel.

For the same data sets, Fig. 5b similarly displays the values of the new variation in taxonomic distinctness index, and relates these to the $95 \%$ limits for the simulated distribution of $\operatorname{VarTD}\left(\Lambda^{+}\right)$from the random subsets of the full UK species list. From the previous section, this can be thought of as adding independent information about the biodiversity structure of these sites, and it is immediately clear that $\Lambda^{+}$separates out the Scilly Isles data sets as distinctive, in a way that is not apparent from the $\Delta^{+}$values. They fall above the

Table 1. Product-moment correlation coefficient, $r$, between paired values of variation in taxonomic distinctness $\left(\Lambda_{m}{ }^{+}\right)$and average taxonomic distinctness $\left(\Delta_{m}{ }^{+}\right)$, calculated from 1000 random subsets of size $m$, the species being drawn (without replacement) from the full species list for British Isles marine nematodes

\begin{tabular}{|ccccccccccccccc|}
\hline 10 & 15 & 20 & 25 & 30 & 40 & 50 & 60 & 80 & 100 & 130 & 160 & 200 & 250 & 300 \\
\hline-0.092 & 0.044 & -0.050 & -0.034 & -0.018 & 0.040 & -0.003 & 0.053 & -0.005 & 0.060 & -0.059 & 0.002 & 0.076 & -0.004 & 0.053 \\
\hline
\end{tabular}


upper limit of the $\Lambda^{+}$funnel, indicating a higher than expected variation in distinctness of species pairs. By analogy with the simple construction in Fig. 2, a possible implication is that there is widespread representation of higher taxa (for the limited number of species found in these algal and sandy shore data sets), and this might have been expected to elevate the AvTD values, but this is counterbalanced by an unevenness in representation of lower taxa. For example, some genera, families or sub-orders may be represented only by single species whilst others are relatively species-rich. The net effect would then be approximately neutral on the average statistic $\Delta^{+}$, but would elevate the variance statistic $\Lambda^{+}$.

The message from the combination of $\Delta^{+}$and $\Lambda^{+}$statistics for the $14 \mathrm{regional} / \mathrm{habitat}$ data sets can be initially seen from a simple scatter plot of the 14 pairs (Fig. 8). The values are comparable in this way, in spite of the differing sampling effort and therefore unequal sizes of the species pools in each study, because the 'Sampling properties' section establishes the almost complete independence of the mean values of $\Delta^{+}$and $\Lambda^{+}$from the species numbers. (Of course the precision of the $\Delta^{+}, \Lambda^{+}$pairs will be a function of $m$, as is clear from the funnel plots of Fig. 5, and the placing of some indication of this precision onto the scatter plot of Fig. 8 is addressed in the next section.) Fig. 8 reveals an interesting characteristic of these data sets: in spite of the virtual absence of an innate (mechanistic) correlation between $\Delta^{+}$and $\Lambda^{+}$(Table 1 ), the observed values of $\Delta^{+}$and $\Lambda^{+}$for these 14 sets are quite strongly linearly

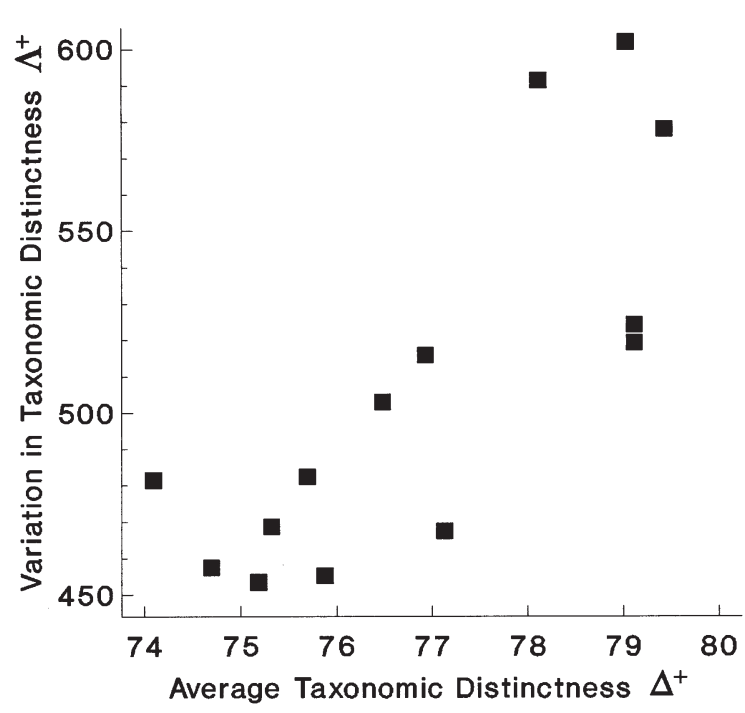

Fig. 8. Scatter plot of VarTD against AvTD values for the real species lists from the $14 \mathrm{regional} / \mathrm{habitat}$ studies. There is a strong observed correlation $(r=0.79)$ which is not internally generated (see Figs. $5 \& 7$ and Table 1 ) and persists even on removal of the 3 Scillies points at the top right of the plot $(r=0.75)$ related, with a product-moment correlation coefficient of $r=0.79(p<0.001)$. This is not simply due to the 3 Scillies points, with their 'near expectation' $\Delta^{+}$and markedly elevated $\Lambda^{+}$values, since on removing these points a significant relationship remains $(r=0.75, \mathrm{p}<$ $0.01)$.

The absence of any mechanistic correlation, arising from the construction of the 2 statistics from the same data sets, allows ecological interpretation of the observed positive correlation. Low values of AvTD for the 'polluted' regions correspond with low values of VarTD. The implication is that pollution is associated with a loss both of the normal wide spread of higher taxa and that the higher taxa lost are those with a relatively simple subsidiary structure, with few branches, such that the higher taxon is represented only by 1 or 2 species, genera or families. This would leave only a limited range of higher taxa, which are all relatively rich in a spread of lower taxa, with a resulting depression both in AvTD and VarTD.

This scenario is entirely consistent with the general interpretation to be found in the literature about the consequences of pollution on the make-up of assemblages: the loss of higher taxa that are represented by rather few species in comparison with the more species-rich taxa. For example, the relative numbers of species in the 4 major taxa of marine macrobenthos generally follow the sequence polychaetes $>$ molluscs $>$ crustaceans $>$ echinoderms, but it is the echinoderms and crustaceans that are reduced first in post-pollution or disturbance situations, followed by the molluscs, with the polychaetes being the most resilient (Warwick \& Clarke 1993). Generally there is an increase in opportunist groups with close taxonomic affinities, which may even sometimes be sibling species or species complexes. For example, certain taxa of benthic marine invertebrates are known to increase dramatically in abundance when levels of particulate organic enrichment become abnormally high (Pearson \& Rosenberg 1978), and have become known as 'pollution indicator' species. There is a strong co-incidence between the taxa involved and those recognised by Knowlton (1993) as comprising sibling species (Warwick \& Clarke 1995). Such taxa include the macrofaunal genera Capitella (Grassle \& Grassle 1976) and Ophryotrocha (Åkesson 1984), and the meiofaunal harpacticoid copepod genus Tisbe (Bergmans 1979, Gee et al. 1985).

\section{Simulation envelopes}

There are potential advantages to assessing the departures of observed values of $\Delta^{+}$and $\Lambda^{+}$from expectation not by separate reference to simulated histograms (such as in Fig. 4), or the 95\% funnel plots they 
generate (Fig. 5), but by combined reference to a 2-dimensional 'envelope' for the simulation pairs $\left(\Delta_{m}{ }^{+}\right.$ $\Lambda_{m}{ }^{+}$) for the relevant number of species $(m)$. One advantage of a bivariate approach is that compensation can automatically be made for the repeated testing inherent in separate reference to 2 univariate simulated distributions. A second advantage is that the rejection region in a 2-dimensional $\left(\Delta^{+}, \Lambda^{+}\right)$plot need not be rectangular, but can be moulded to account for any mechanistic correlation between $\Delta_{m}{ }^{+}$and $\Lambda_{m}{ }^{+}$. Thus, if random selections from a master list showed that $\Delta_{m}{ }^{+}$and $\Lambda_{m}{ }^{+}$were expected to be positively correlated (which, while not the case for the UK marine nematode data, could well be true for other master lists), then rejection could be indicated by moderately low AvTD combined with moderately high VarTD (or vice versa), when neither value is low or high enough to imply rejection in the separate tests.

Simulations of $\left(\Delta_{m}{ }^{+}, \Lambda_{m}^{+}\right)$pairs from the nematode master list, viewed as a scatter plot, have already been seen for the case $m=100$ (Fig. 7). Superimposed on Fig. 7 are the real data points for the Scillies (all habitats), Clyde 1 and Liverpool Bay, which all have approximately 100 species (102, 112 and 97 respectively). In their different ways, all 3 clearly lie outside the 'region of experience' for the simulated values. This heuristic comparison can be fomalised by fitting a bivariate distribution to the simulated values and defining a contour of that density function enclosing $95 \%$ of the probability. The null hypothesis - that the observed $\left(\Delta^{+}, \Lambda^{+}\right)$pair has taxonomic structure representative of a random sample from the master list-is rejected (on a $5 \%$ level test) if this point falls outside the $95 \%$ envelope.

Although the distribution of $\Lambda_{m}{ }^{+}$in Fig. $4 \mathrm{~b}$ does not appear to be skewed, there is clearly some asymmetry in the $\Delta_{m}{ }^{+}$distribution of Fig. 4a. Looking across the simulations for a full range of $m(=10,15,20, \ldots, 250)$ the same features are evident: whilst $\Lambda_{m}{ }^{+}$appears to be not too far from normality, $\Delta_{m}{ }^{+}$can be markedly leftskewed, especially for the smaller values of $\mathrm{m}$. A power transformation combined with a location shift and reversal:

$$
Y^{*}=(a-Y)^{b}
$$

can be used to transform the marginal distribution of $Y=\Delta_{m}{ }^{+}$(and $\Lambda_{m}{ }^{+}$) to approximate symmetry. Separately for each $m$, this optimises over the parameters a and $b$, where $a$ is only permitted to take positive values greater than the maximum simulated $Y$ but $b$ can be any positive number, greater or less than 1 corresponding to corrections for, respectively, right or leftskewness in the original distribution of $Y$. Values of $a$ and $b$ are chosen to minimise the standardised third moment, $\mu_{3} /\left(\mu_{2}\right)^{3 / 2}$ (Kendall \& Stuart 1963). A bivariate normal distribution, including a correlation term, is then fitted to the 2 transformed $\left(\Delta_{m}{ }^{+}, \Lambda_{m}{ }^{+}\right)$variables, simply by equating the sample means, variances and correlation for the transformed variables with their population equivalents. Contours enclosing specific probabilities (e.g. 95, 90, 75, 50\%) are back-transformed to the original scales and viewed superimposed on the original scatter plot of simulated values. This is seen in Fig. 9, for the case of $m=100$ species, and the fit is clearly excellent for practical purposes: 26 of the 500 simulations (5.2\%) fall outside the $95 \%$ contour, and $53(10.6 \%)$ fall outside the $90 \%$ contour, with these values being fairly evenly spread around the (backtransformed) ellipses. Although not shown, the fits are also perfectly adequate for other values of $m$. As anticipated, for fitting the AvTD marginal distribution, optimum values of the transformation power $b$ range from as severe as 0.1 or even 0.05 to a milder 0.4 , indicating substantial left-skewness for all values of $m$. By contrast, the fits for VarTD require powers not much different than 1 (at most 2), implying only mild rightskewness, if any at all.

Although computationally straightforward to automate robustly, the fitting procedure is an ad hoc one: lack of skewness in the 2 marginal distributions does not in general imply univariate normality let alone bivariate normality of the 2 variables in combination. A

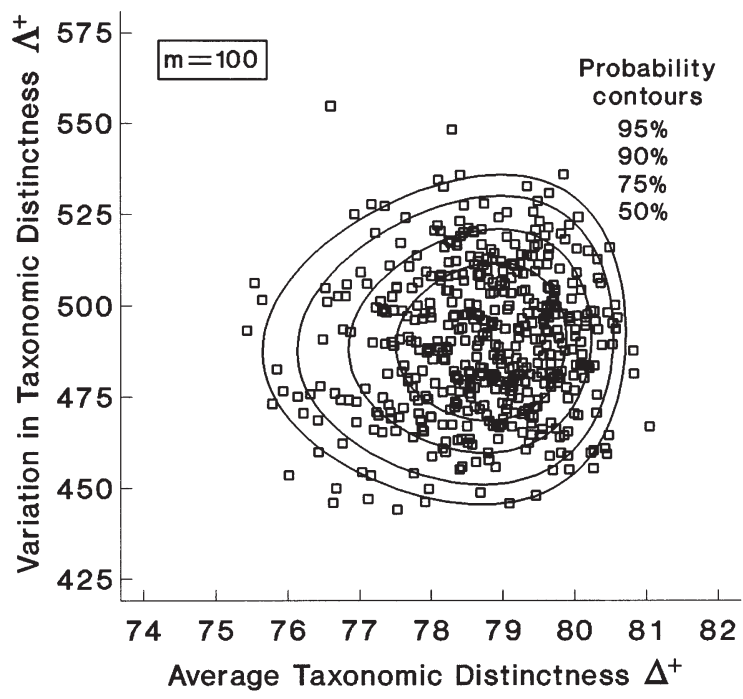

Fig. 9. Scatter plot of simulated VarTD, AvTD pairs, as in Fig. 7 (i.e. for $m=100$ species selected from the full UK list), but with superimposed probability contours. For example, $95 \%$ of simulated values would be expected to fall within the outer envelope, $90 \%$ inside the second outermost, etc. (94.8 and $89.4 \%$ do so, respectively, in this case). Contours are back-transformed from bivariate normal ellipses fitted on a locationshifted (reversed) power transform scale (text Eq. 8), selected separately for each axis to minimise skewness of the simulated values 


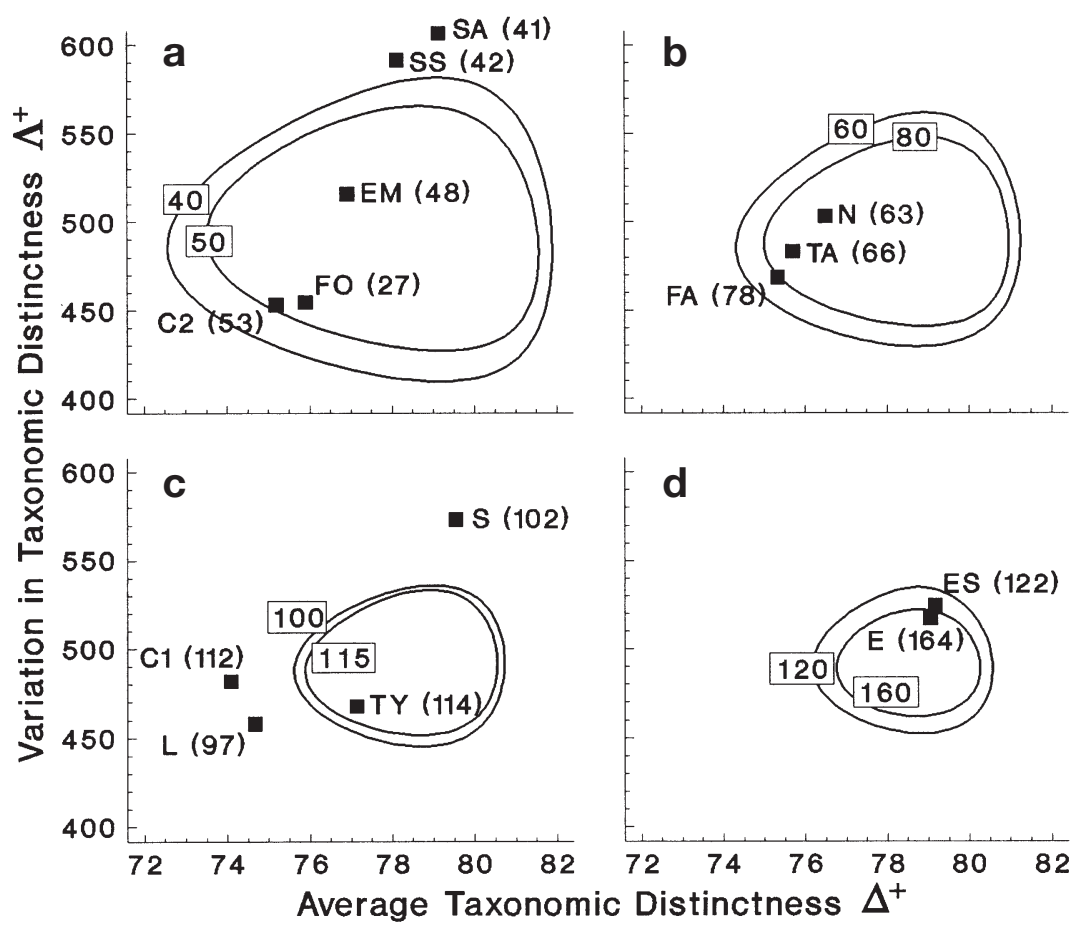

Fig. 10. Fitted $95 \%$ probability contours of the joint VarTD, AvTD distributions, from 1000 simulations, as given in Fig. 9 but for a range of values of $m$, the number of species. For greater clarity, these are separated into 4 plots $\left(a-d_{i} m=40,50\right.$; 60,$80 ; 100,115$; and 120,160 , respectively), to allow the real $\Lambda^{+}, \Delta^{+}$pairs for the 14 regional/habitat data sets ( $\bullet$, size of species list in parentheses) to be superimposed on a contour plot for appropriate values of $m$. Data points outside the relevant $95 \%$ contour imply statistical evidence of 'departure from expectation' for those studies. The decreasing area, but stable location, of these contours as $m$ increases is the equivalent for this joint distribution of the separate probability funnel plots of Fig. 5 (the 'marginal' distributions of $\Delta^{+}$and $\Lambda^{+}$). Abbreviations as in Fig. 3

proper statistical treatment via transformations, which would potentially also cater for kurtosis effects, would be given by the maximum likelihood approach of Box \& Cox (1964), but the extra sophistication and potential complications of this formal approach seem unwarranted here, given the pragmatic adequacy of the envelopes generated by minimising the marginal skewness terms.

Finally then, Fig. 10 displays the outcome of constructing the $95 \%$ probability envelopes for subsamples from the master list of British Isles marine nematode species, for increasing values of the number of species, $m$. These could be displayed on a single $\left(\Delta^{+}, \Lambda^{+}\right)$plot, but to improve clarity they have been divided into 4 component plots showing cases: $m=40$ and 50 (Fig. 10a); $m=60$ and 80 (Fig. 10b); $m=100$ and 115 (Fig. 10c); and $m=120$ and 160 (Fig. 10d). These $m$ values are chosen to cover the range of sizes of the 14 regional/habitat lists, whose observed $\left(\Delta^{+}, \Lambda^{+}\right)$values are superimposed on the appropriate component plot (the size of their lists are also indicated, in parentheses in
Fig. 10). Each observed value should be compared with its relevant envelope, or the interpolation of the contours from the 2 bracketing values of $m$. This, therefore, facilitates a simple assessment of the status of these samples.

The Northumberland (N), Tamar (TA), Tyne (TY) and all the Exe (E, ES, EM) lists give average and variation in taxonomic distinctness values which fall within their respective envelopes, indicating no significant difference in taxonomic structure from that for the British Isles list as a whole. The same is true of the data from the Forth (FO), although because of the low number of species recorded $(m=27)$ the test will have limited statistical power to detect a decrease in AvTD and/or VarTD, both of which appear somewhat depressed in this case. The data from the Clyde (C2, Jayasree study) and the Fal (FA) are both on the borderline of significance, again in the direction of decreased $\Delta^{+}$and $\Lambda^{+}$, and the remaining studies all show clearly significant departure from the null hypothesis. For the Clyde (C1, Lambshead study) and Liverpool Bay, this is primarily in the direction of decreased AvTD, although also with a suggestion of low VarTD for the Liverpool data.

For all 3 Scillies data sets (S, SA, SS), the pattern is quite different, with a pronounced increase in VarTD, though an 'expected' level of AvTD. The distinctively high values of VarTD for the Scillies may result from the reduced habitat diversity. One major nematode habitat, fine silty sediments, is absent. There are no rivers or estuaries, which are sources of fine sediment in mainland coastal waters. The sea is exceptionally clear here; kelp (Laminaria ochroleuca), for example, occurs at depths of $30 \mathrm{~m}$, the deepest in the UK. This means that there is no fine sediment associated with secondary habitats such as seaweeds and their holdfasts. The proportions of the 3 main nematode groups, enoplids:chromadorids:monhysterids, is $43.1: 36.3: 20.6$, compared with average values for the complete British fauna of 26.5:46.2:27.3. Enoplids favour clean habitats, and are not at all characteristic of sedimentary habitats (Warwick et al. 1998), whereas monhysterids are more characteristic of fine sediments. The relative overrepresentation of the former and under-representation of the latter is thus commensurate with the absence of fine sedimentary habitats. 


\section{Concluding remarks}

This paper has presented a simpler and more elegant way of eliciting unevenness in the structure at different levels of a taxonomic or phylogenetic tree than the method of Clarke \& Warwick (1999), which recalculated AvTD whilst successively compressing the higher taxonomic levels. Note, though, that the latter method also revealed the distinctive nature of the Scillies data. One can speculate that this combination of an expected $\Delta^{+}$ value with a greater than expected $\Lambda^{+}$may be characteristic of an island fauna, if our earlier surmise is correct and the elevated VarTD is due to a reduction in habitat diversity. Theories of island biogeography have largely been developed from data on species that are easily censused and for which complete inventories can be produced in relation to island size, such as birds, reptiles and certain groups of insects (MacArthur \& Wilson 1967). For groups such as free-living nematodes, or other small cryptic taxa, a complete census is rarely possible, except for very small areas, and the list for the Scillies archipelago is certainly not complete-increased sampling effort would undoubtedly reveal more. AvTD and VarTD, with their appealing sampling properties, therefore offer a useful alternative, and might also help to address longstanding questions concerning island biogeography that cannot be resolved by a count of the number of species alone: for example whether increasing numbers of species are a function of increasing island size per se, or are related to the larger number of habitats.
Because of the impracticality of routinely attempting comprehensive surveys, surrogacy methods will clearly become the norm in biodiversity estimation (Harper \& Hawksworth 1994), and the search for appropriate indicators of marine and coastal biodiversity has become an important research goal (Feral 1999). Surrogates might take the form of a subset of easily censused species from the total biota, but the crucial issue then concerns how valid a measure this surrogate provides of the true total biodiversity. The species richness of the surrogate could never be expected to be representative and, here again, AvTd and VarTD could play a useful role. On a regional rather than a single habitat basis, a census of all living species from the whole spectrum of habitat types would be very time-consuming and costly. The concept of sampling a single spatially averaged subset of species such as a death assemblage (e.g. of molluscs), as a surrogate for regional biodiversity, is therefore appealing; but this would be dependent on that assemblage being representative of the full biodiversity, and AvTD and VarTD could provide appropriate measures of this representativeness. Such ideas will be pursued in a subsequent paper.

Acknowledgements. This work forms part of the Biodiversity Core Strategic Research Programme of the Natural Environment Research Council's Plymouth Marine Laboratory, UK, and was also part-funded by the UK Ministry of Agriculture, Fisheries and Food (project no. AE1113). We are grateful to the anonymous referees for their encouraging comments.

Appendix A. General expression for mean of VarTD

For a subset of $m$ species drawn at random from the full set of $s$ species, the AvTD statistic is defined as

$$
\Delta_{m}^{+}=\left[\Sigma \Sigma_{i \neq j} \omega_{i j} Y_{i} Y_{j}\right] /[m(m-1)]
$$

where the double summation is over all $i, j=1, \ldots s$ species $(i \neq j)$ and $Y_{i}$ is an indicator random variable determining whether the $i$ th species is selected $\left(Y_{i}=1\right)$ or not $\left(Y_{i}=0\right)$. Clarke \& Warwick (1998) show that

$$
\mathrm{E}\left(\Delta_{m}{ }^{+}\right)=\Delta^{+}
$$

the AvTD value for the full set of $s$ species, and

$$
\begin{aligned}
\operatorname{var}\left(\Delta_{m}{ }^{+}\right)= & {[\{2(s-m)\} /\{m(m-1)(s-2)(s-3)\}] } \\
& \times\left[(s-m-1) \sigma_{\omega}{ }^{2}+2(s-1)(m-2) \sigma_{\bar{\omega}}^{2}\right]
\end{aligned}
$$

where

$$
\begin{aligned}
\sigma_{\omega}^{2} & =\left[\left\{\Sigma \Sigma_{l \neq j} \omega_{i j}{ }^{2}\right\} /\{s(s-1)\}\right]-\bar{\omega}^{2} \equiv \Lambda^{+} \\
\sigma_{\bar{\omega}}^{2} & =\left[\left(\Sigma_{i} \bar{\omega}_{i}^{2}\right) / s\right]-\bar{\omega}^{2} \\
\bar{\omega}_{i} & =\left(\Sigma_{j(\neq i)} \omega_{i j}\right) /(s-1) \\
\bar{\omega} & =\left(\Sigma_{i} \bar{\omega}_{i}\right) / s=\left[\Sigma \Sigma_{i \neq j} \omega_{i j}\right] /[s(s-1)] \equiv \Delta^{+}
\end{aligned}
$$

For the same subset of $m$ species, the VarTD statistic and its expectation are, respectively:

$$
\begin{aligned}
\Lambda_{m}{ }^{+} & =\left[\left\{\Sigma \Sigma_{i \neq j} \omega_{i j}{ }^{2} Y_{i} Y_{j}\right\} /\{m(m-1)\}\right]-\left(\Delta_{m}{ }^{+}\right)^{2} \\
\mathrm{E}\left(\Lambda_{m}{ }^{+}\right) & =\left[\left\{\Sigma \Sigma_{i \neq j} \omega_{i j}{ }^{2} \mathrm{E}\left(Y_{i} Y_{j}\right)\right\} /\{m(m-1)\}\right]-\mathrm{E}\left\{\left(\Delta_{m}{ }^{+}\right)^{2}\right\}
\end{aligned}
$$

and using the relationships

$$
\begin{aligned}
& \mathrm{E}\left(Y_{i} Y_{j}\right)=\operatorname{Pr}\left\{Y_{i}=1, Y_{j}=1\right\}=[m(m-1)] /[s(s-1)] \\
& \mathrm{E}\left\{\left(\Delta_{m}^{+}\right)^{2}\right\}=\operatorname{var}\left(\Delta_{m}^{+}\right)+\left[\mathrm{E}\left(\Delta_{m}^{+}\right)\right]^{2}
\end{aligned}
$$

it follows from Eq. (A2), and Eqs. (3) \& (4) of the main text, that

$$
\begin{aligned}
\mathrm{E}\left(\Lambda_{m}{ }^{+}\right) & =\left[\left\{\Sigma \Sigma_{i \neq j} \omega_{i j}{ }^{2}\right\} /\{s(s-1)\}\right]-\left[\operatorname{var}\left(\Delta_{m}^{+}\right)+\left(\Delta^{+}\right)^{2}\right] \\
& =\Lambda^{+}-\operatorname{var}\left(\Delta_{m}^{+}\right)
\end{aligned}
$$

Since $\operatorname{var}\left(\Delta_{m}{ }^{+}\right) \geq 0$, the bias in estimation of $\Lambda^{+}$from a random subsample will always be negative but Eq. (A3) shows that it will generally be very small. For example, from Eq. (A4) it is clear that the coefficient of $\sigma_{\omega}^{2}\left(\equiv \Lambda^{+}\right)$in (A3) is roughly $2 / \mathrm{m}^{2}$, when $s$ is large and $m$ is modest (the typical situation). This therefore contributes a percentage bias of approximately $200 / \mathrm{m}^{2}$, which is already minor $(2 \%)$ for $m$ as small as 10 , and becomes entirely negligible for $m$ much larger than this. It is harder to generalise about the other bias term (in $\sigma_{\bar{\omega}}^{2}$ ), since it is not directly proportional to $\Lambda^{+}$, but Eq. (A5) shows that it reflects the level of asymmetry in the complete tree (a fully symmetric tree would give $\sigma_{\bar{\omega}}^{2}=0$ ). In practice, it appears that this second bias term can be the larger of the two, but is still essentially negligible. For the British Isles nematode list of 395 species, the 2 bias terms constitute only approximately a $1 \%$ underestimation of $\Lambda^{+}$ for a subsample size $m$ of 27 (the lowest value of $m$ for any of the 14 regionally/habitat-defined data sets). 
Appendix B. Special case of only 3 hierarchical levels

In the case where there are only 2 steps (3 levels) in the hierarchical classification, say species within genera within a single family, let $g$ denote the number of distinct genera and $\left\{s_{k i} k=1, \ldots g\right\}$ the number of species in each genus $\left(\Sigma_{k} S_{k}=s\right)$. In the general case where the path lengths are denoted by $\omega_{1}$ (species in the same genus) and $\omega_{2}$ (species in different genera), there are

$$
A=\Sigma_{k} s_{k}\left(s_{k}-1\right) / 2
$$

path lengths of $\omega_{1}$, with the remainder of the

$$
B=s(s-1) / 2
$$

paths being of length $\omega_{2}$. From Eq. (1) of the main text, AvTD is therefore

$$
\Delta^{+}=\left[\omega_{1} A+\omega_{2}(B-A)\right] / B
$$

and, from Eqs. (3) \& (4) of the main text, VarTD is

$$
\Lambda^{+}=\left\{\left[\omega_{1}^{2} A+\omega_{2}^{2}(B-A)\right] / B\right\}-\left(\Delta^{+}\right)^{2}
$$

Rearranging Eqs. (B3) \& (B4) as, respectively

$$
\begin{gathered}
\left(\omega_{2}-\omega_{1}\right)(A / B)=\omega_{2}-\Delta^{+} \\
\Lambda^{+}=\left[\omega_{2}^{2}-\left(\omega_{2}+\omega_{1}\right)\left(\omega_{2}-\omega_{1}\right)(A / B)\right]-\left(\Delta^{+}\right)^{2}
\end{gathered}
$$

one can substitute Eq. (B5) into (B6) to yield $\Lambda^{+}$as a quadratic in $\Delta^{+}$:

$$
\begin{aligned}
\Lambda^{+} & =-\omega_{1} \omega_{2}+\left(\omega_{1}+\omega_{2}\right) \Delta^{+}-\left(\Delta^{+}\right)^{2} \\
& =\left(\omega_{2}-\Delta^{+}\right)\left(\Delta^{+}-\omega_{1}\right)
\end{aligned}
$$

Eq. (7) of the main text cites this equation in the standard case of equal step lengths, $\omega_{1}=50, \omega_{2}=100$, but (B7) shows that, for any path length weights in a 3-level hierarchy, VarTD will always be a simple quadratic in AvTD. The approximate independence of $\Delta_{m}{ }^{+}$and $\Lambda_{m}{ }^{+}$, observed for subsamples drawn from the 7-level hierarchy of British marine nematodes is, therefore, not a generalisable result. Clearly there are simple situations in which VarTD gives no additional information to that provided by AvTD, although it seems a plausible hypothesis that the richer the problem is (i.e. the more highly structured the list in terms of number of levels), the greater the likelihood that it will be informative to examine both statistics.

\section{LITERATURE CITED}

Åkesson B (1984) Speciation in the genus Ophryotrocha (Polychaeta, Dorvilleidae). Fortsch Zool 29:299-316

Austen MC, Warwick RM (1989) Comparison of univariate and multivariate aspects of estuarine meiobenthic community structure. Estuar Coast Shelf Sci 29:23-42

Bergmans M (1979) Taxonomic notes on species of Tisbe (Copepoda: Harpacticoida) from a Belgian sluice dock. Zool Scr 8:211-220

Box GEP, Cox DR (1964) An analysis of transformations. J R Statist Soc Ser B 26:211-243

Clarke KR, Warwick RM (1998) A taxonomic distinctness index and its statistical properties. J Appl Ecol 35:523-531

Clarke KR, Warwick RM (1999) The taxonomic distinctness measure of biodiversity: weighting of step lengths between hierarchical levels. Mar Ecol Prog Ser 184:21-29

Faith DP (1992) Conservation evaluation and phylogenetic diversity. Biol Conserv 61:1-10

Faith DP (1994) Phylogenetic pattern and the quantification of organismal biodiversity. Philos Trans R Soc Lond Ser B Biol Sci 345:45-58

Feral JP (1999) Indicators of marine and coastal biodiversity of the Mediterranean Sea. United Nations Environment Programme, Tunis

Gee JM, Warwick RM, Schanning M, Berge JA, Ambrose Jr WG (1985) Effects of organic enrichment on meiofaunal abundance and community structure in sublittoral soft sediments. J Exp Mar Biol Ecol 91:247-262

Grassle JP, Grassle JF (1976) Sibling species in the marine pollution indicator species Capitella (Polychaeta). Science 192:567-569

Hall SJ, Greenstreet SP (1998) Taxonomic distinctness and diversity measures: responses in marine fish communities. Mar Ecol Prog Ser 166:227-229

Harper JL, Hawksworth DL (1994) Biodiversity: measurement and estimation. Philos Trans R Soc Lond Ser B Biol Sci: 345:5-12

Humphries CJ, Williams PH, Vane-Wright RI (1995) Measuring biodiversity value for conservation. Annu Rev Ecol Syst 26:93-111
Jayasree K (1976) Systematics and ecology of free-living marine nematodes from polluted intertidal sand in Scotland. $\mathrm{PhD}$ thesis, University of Aberdeen

Kendall MG, Stuart A (1963) The advanced theory of statistics, Vol 1. Griffin, London

Knowlton N (1993) Sibling species in the sea. Annu Rev Ecol Syst 24:189-216

Lambshead PJD (1986) Sub-catastrophic sewage and industrial waste contamination as revealed by marine nematode faunal analysis. Mar Ecol Prog Ser 29:247-260

Lorenzen S (1981) Entwurf eines phylogenetischen Systems der freilebenden Nematoden. Veröff Inst Meeresforsch Bremerhav, Suppl 7:1-449

Lorenzen S (1994) The phylogenetic systematics of freeliving nematodes. Ray Society, London

MacArthur RH, Wilson EO (1967) The theory of island biogeography. Princeton University Press, Princeton, NJ

May RM (1990) Taxonomy as destiny. Nature 347:129-130

Nee S, May RM (1997) Extinction and the loss of evolutionary history. Science 278:692-694

Pearson TH, Rosenberg R (1978) Macrobenthic succession in relation to organic enrichment and pollution of the marine environment. Oceanogr Mar Biol Annu Rev 16:229-311

Piepenburg D, Voss J, Gutt J (1997) Assemblages of sea stars (Echinodermata: Asteroidea) and brittle stars (Echinodermata: Ophiuroidea) in the Weddell Sea (Antarctica) and off Northeast Greenland (Arctic): a comparison of diversity and abundance. Polar Biol 17:305-322

Platt HM, Warwick RM (1983) Freeliving marine nematodes. Part I. British enoplids. Synop Brit Fauna New Ser 28: $1-307$

Platt HM, Warwick RM (1988) Freeliving marine nematodes. Part II. British Chromadorida. Synop Brit Fauna New Ser 38:1-502

Price ARG, Keeling MJ, O'Callaghan CJ (1999) Ocean-scale patterns of 'biodiversity' of Atlantic asteroids determined from taxonomic distinctness and other measures. Biol J Linn Soc 66:187-203

Rogers SI, Clarke KR, Reynolds JD (1999) The taxonomic distinctness of coastal bottom-dwelling fish communities of the North-east Atlantic. J Anim Ecol 68:769-782 
Somerfield PJ, Gee JM, Widdicombe S (1993) The use of meiobenthos in marine pollution monitoring programmes. Plymouth Marine Laboratory, Plymouth, UK (Misc Publ LIB-33A+LIB-33B)

Somerfield PJ, Gee JM, Warwick RM (1994a) Soft sediment meiofaunal community structure in relation to a long-term heavy metal gradient in the Fal estuary system. Mar Ecol Prog Ser 105:79-88

Somerfield PJ, Gee JM, Warwick RM (1994b) Benthic community structure in relation to an instantaneous discharge of waste water from a tin mine. Mar Pollut Bull 28:363-369

Somerfield PJ, Rees HL, Warwick RM (1995) Interrelationships in community structure between shallow-water marine meiofauna and macrofauna in relation to dredgings disposal. Mar Ecol Prog Ser 127:103-112

Vane-Wright RI, Humphries CJ, Williams PH (1991) What to protect? Systematics and the agony of choice. Biol Conserv 55:235-254

Warwick RM (1971) Nematode associations in the Exe estuary. J Mar Biol Assoc UK 51:439-454

Editorial responsibility: Otto Kinne (Editor),

Oldendorf/Luhe, Germany
Warwick RM, Buchanan JB (1970) The meiofauna off the coast of Northumberland. I. The structure of the nematode population. J Mar Biol Assoc UK 50:129-146

Warwick RM, Clarke KR (1993) Comparing the severity of disturbance: a meta-analysis of marine macrobenthic community data. Mar Ecol Prog Ser 92:221-231

Warwick RM, Clarke KR (1995) New 'biodiversity' measures reveal a decrease in taxonomic distinctness with increasing stress. Mar Ecol Prog Ser 129:301-305

Warwick RM, Clarke KR (1998) Taxonomic distinctness and environmental assessment. J Appl Ecol 35:532-543

Warwick RM, Coles JW (1977) The marine flora and fauna of the Isles of Scilly. Free-living Nematoda. J Nat Hist 11: 393-407

Warwick RM, Platt HM, Somerfield PJ (1998) Freeliving marine nematodes. Part III. British Monhysterida. Synop Brit Fauna 53:1-296

Williams PH, Humphries CJ, Vane-Wright RI (1991) Measuring biodiversity: taxonomic relatedness for conservation priorities. Aust Syst Bot 4:665-679

Submitted: April 19, 2000; Accepted: September 29, 2000 Proofs received from author(s): June 5, 2001 